\title{
Electrocardiogram in apical hypertrophic cardiomyopathy with a speculation as to the mechanism of its features
}

\author{
J. E. Madias
}

Published online: 18 May 2013

(C) The Author(s) 2013. This article is published with open access at Springerlink.com

\begin{abstract}
A variety of electrocardiogram (ECG) alterations in patients with apical hypertrophic cardiomyopathy (AHCM) have been described in the literature, but no relevant quantitative analysis has been provided; thus the objective of this communication was to review the relevant literature and using two cases of patients with AHCM, to provide such a quantitative analysis. Using PubMed to search the literature 13 studies on the ECG in patients with AHCM were identified and evaluated; also a quantitative analysis of the ECG attributes in two patients was carried out. Qualitative ECG features from the literature on patients with AHCM is discussed. Also a description of the ECG in two patients with AHCM has identified as typical features truly giant QRS complexes in the precordial leads, particularly in lead V4, with rightward superior, and posterior shift of the T-wave vector, the latter being a newly described ECG correlate of AHCM. A speculation as to the possible mechanism of the observed ECG features is included.
\end{abstract}

Keywords Electrocardiogram · Electrophysiology · Hypertrophic cardiomyopathy · Apical hypertrophic cardiomyopathy · Giant R-waves · Giant T-waves

\section{Introduction}

The impetus for this communication was the author's recent encounter at the Cardiac Clinic (CC) with two patients with apical hypertrophic cardiomyopathy (AHCM), with almost identical electrocardiograms (ECGs).

\section{J. E. Madias}

Mount Sinai School of Medicine, New York University,

New York, NY, USA

\section{J. E. Madias $(\bowtie)$}

Division of Cardiology, Elmhurst Hospital Center,

79-01 Broadway,

Elmhurst, NY 11373, USA

e-mail: madiasj@nychhc.org

\section{Case reports}

Case \#1 A 59-year-old South Asian man with a history of nonobstructive coronary artery disease, diagnosed by cardiac catheterisation, hypertension, diabetes mellitus, 40-pack year cigarette smoking, and severe peripheral arterial disease involving his aorta, iliac and carotid arteries is being presented. He had been followed at our $\mathrm{CC}$ for the past 5 months. His echocardiogram (ECHO) 4 months prior to the ECG recording of Fig. 1 showed AHCM with increased thickness of the left ventricular wall at the apex, sparing of the free walls, with hypokinesis of the basal inferior, midinferior, and basal inferolateral segments, and a left ventricular ejection fraction of $45 \%$.

Case \#2 A 50-year-old South Asian man has been followed at our CC for the past 8 years for AHCM. His ECHO, two weeks after the ECG of Fig. 2, showed AHCM, consisting of left ventricular wall thickness most prominent at the apex and mid-cavity region, with hyperdynamic left ventricular contractility, an ejection fraction of $70 \%$, and mid-cavitary left ventricular obliteration. This patient had three episodes of syncope attributed to vasovagal mechanism, with two of them related to micturition. His brother had died suddenly at the age of 40 of an unexplained cause. The patient had had an automobile accident with injuries involving his left shoulder, knee, and spine, for which he is awaiting surgery, and following which we are pursuing implantation of an automatic cardioverter/defibrillator, about which he has not agreed as yet.

\section{Automated ECG analysis}

The automated interpretation of the printed reports of the ECGs (Figs. 1 and 2) included sinus rhythm, left ventricular hypertrophy (LVH) left ventricular anterolateral repolarisation abnormalities due to $\mathrm{LVH}$, and probable ischaemia. 
Fig. 1 ECG of patient \#1;

analysis is provided in the text

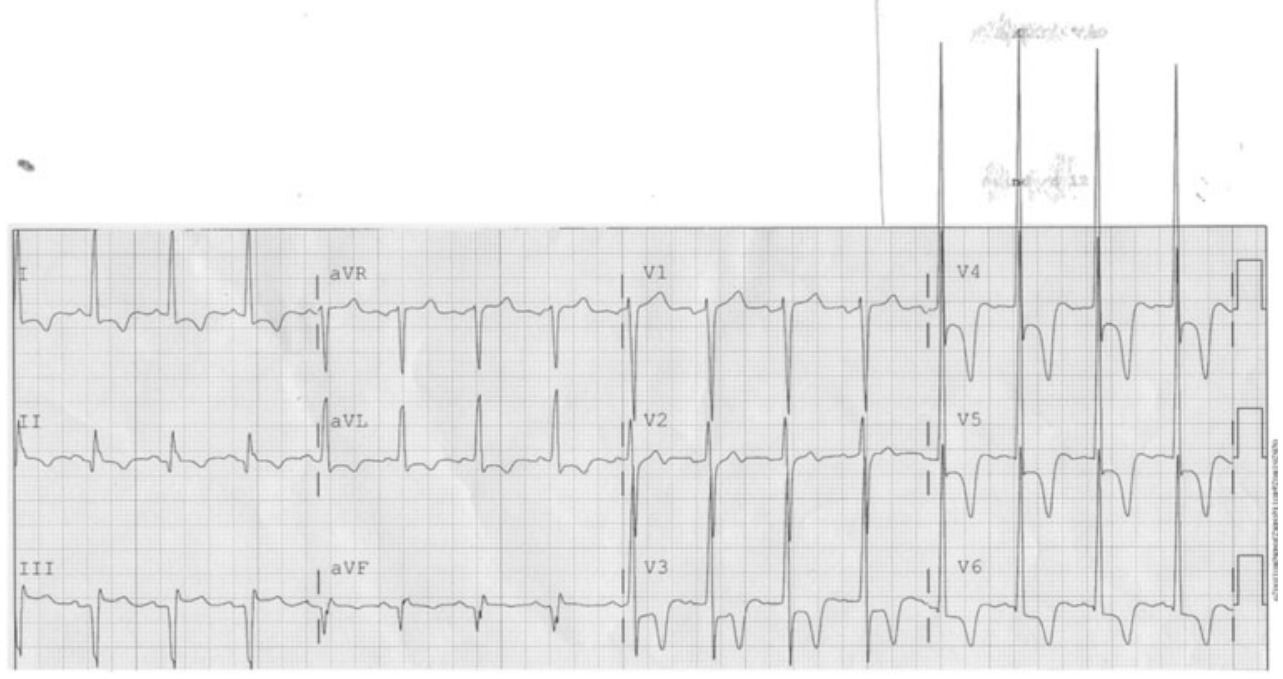

This prompted the author to review the literature on the ECG of AHCM, and to analyse the two ECGs quantitatively, with the intention of stimulating other colleagues who have access to large databases and registries of patients with AHCM to take another look at the ECGs of such patients for possible distinctive features specific for AHCM.

\section{Author's ECG analysis}

Both ECGs (Figs. 1 and 2) showed an inverted, sometimes giant $(>1.0 \mathrm{mV}) \mathrm{T}$ wave in all 12 leads, except aVR and V1; patient \#1 also had an upright T wave in III, and Q waves in II, III, and aVF, simulating an inferior myocardial infarction. Quantitative analyses of the 2 ECGs are shown on Table 1. Giant $\mathrm{R}$ waves in the precordial leads, particularly V4 (Figs. 1 and 2), were noted. Patient \#1 had two ECGs 4 1/ 2 months apart, with some change in the above; patient \#2 had four ECGs over the course of 8 years and 3 months. In comparing these ECGs it appeared that some variation in the ST depression and T-wave inversion was noted mostly in the precordial leads, and could partially be accounted for by the variation in the placement of the thoracic recording sites for the V1-V6 leads in the serial ECGs. The most impressive consistent changes in AHCM appear to be the enormity of the amplitude of the QRS complexes, mainly the R waves of the precordial leads, particularly V4 (Figs. 1 and 2) and the rightward posterior and superior axis of the $\mathrm{T}$ waves.

\section{Discussion}

Case \#1 reveals that patients with AHCM are not immune to other illnesses such as atherosclerosis involving the coronary and peripheral arteries, and such comorbidities should be sought during the follow-up for AHCM. Patient \#1 had comorbidities partially confounding the features of AHCM; however the influence, from the ECG point of view, of the features attributed to AHCM (enormous QRS, particularly R waves and large inverted $\mathrm{T}$ waves with specific threedimensional orientation) were overwhelming. The mechanism of these two ECG features is attributed to the location
Fig. 2 ECG of patient \#2; analysis is provided in the text

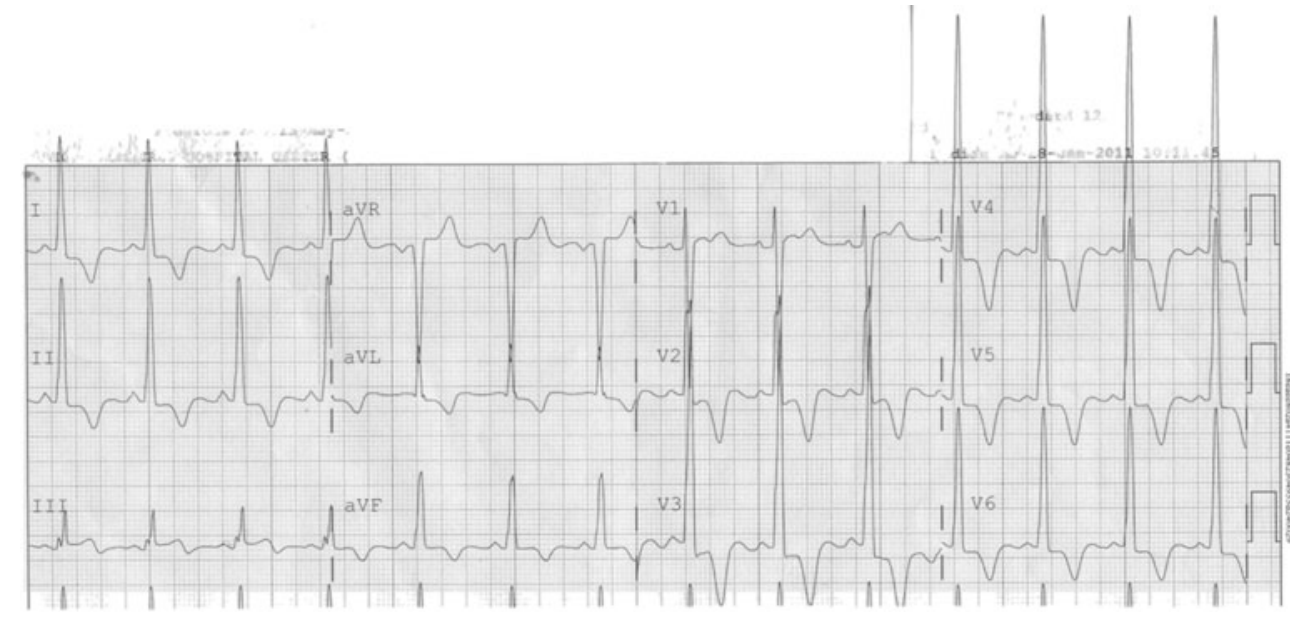


Table 1 Quantitative ECG analysis of 2 patients with apical hypertrophic cardiomyopathy, based on the automated measurements of the ECG management system

\begin{tabular}{|c|c|c|c|c|c|c|c|c|c|c|}
\hline FQRSA & HQRSA & FTA & HTA & Tallest QRSAmp & $\Sigma$ QRSAmpLbLds & $\Sigma \mathrm{QRSPr}$ & $\Sigma$ QRS12Lds & QRSd & Tallest T & QTc \\
\hline-14 & 6 & 178 & 197 & 6.245 & 8.201 & 23.882 & 32.083 & 98 & 1.595 & 473 \\
\hline 45 & 29 & 200 & 214 & 4.818 & 10.723 & 21.749 & 32.472 & 104 & 1.293 & 522 \\
\hline
\end{tabular}

FQRSA frontal QRS axis in degrees; HQRSA horizontal QRS axis in degrees; FTA frontal T axis in degrees; HTA horizontal T axis in degrees; tallest QRSAmp tallest QRS amplitude in $\mathrm{mV} ; \Sigma Q R S A m p L b L d s$ sum of QRS amplitudes of the 6 limb leads in $\mathrm{mV}$; $\Sigma Q R S P r$ sum of QRS amplitudes of the 6 precordial leads in $\mathrm{mV}$; $\Sigma Q R S 12 \mathrm{Lds}$ sum of QRS amplitudes of the $12 \mathrm{ECG}$ leads in $\mathrm{mV}$; QRSd QRS duration in ms; tallest T tallest T-in $\mathrm{mV}$; QTc corrected QT in $\mathrm{ms}$

of hypertrophy in patients with AHCM. Specifically, hypertrophy involving the left ventricular septal, anterior, lateral and inferior, midventricular, and/or basal territories is mitigated by cancellation of depolarisation forces generated by normal or hypertrophic regions; on the contrary, the apical distribution of hypertrophy in patients with AHCM opposite to the nonmuscular elements of the fibrous cardiac skeleton of the mitral valve and annular plane provides the essentials for an unopposed expression of depolarisation vectorial forces directed towards the apex resulting in enormous $\mathrm{R}$ waves. The enormity of the R waves in patients with AHCM may also be related to histological changes (disarray of myofibrils and fibrosis) inherent to hypertrophic cardiomyopathy. Such changes may enhance the resistivity of the cardiac muscle which thus produces an increase in the amplitude of the $\mathrm{R}$ waves. A parallel which comes to mind is the frequent occurrence of very large $\mathrm{R}$ waves in glycogen storage disease type II, also known as Pompe disease or acid maltase deficiency, in which the large amplitude of $\mathrm{R}$ waves has been attributed to an increase in the resistivity of myocardial tissue due to accumulation of glycogen in the lysosomes and associated fibrosis. ST-segment and T-wave secondary changes usually mirror in extent the amplitude of QRS complexes and $\mathrm{R}$ waves in all forms of cardiac ventricular hypertrophy. Thus, it should be expected that the large $\mathrm{R}$ waves are associated with large secondary T-wave changes with opposite vectorial orientation, producing 'giant' T waves directed opposite the cardiac apex, i.e., superiorly, rightwards and posteriorly, as observed in these two patients with AHCM.

The ECG features of AHCM, which is the focus of this communication, are reported to be giant negative $\mathrm{T}$ waves $[1,2]$, ST depression and negative U waves in II, III, aVF, V4-V6, and a prolonged QTc [1]. However, giant negative T waves were not invariably seen in AHCM [2]. Also the depth of the negative T waves and ST-segment depression has been reported to vary from hour to hour, day to day, and year to year [1]. Others have described increased S-wave voltage in $\mathrm{V} 1+\mathrm{R}$-wave voltage in $\mathrm{V} 5$ up to $8.1 \mathrm{mV}$ with inverted $\mathrm{T}$ waves, both of which either remained stable, or increased or decreased overtime in some patients [3, 4], or stable ECGs, increase or decrease by $10 \mathrm{~mm}$ or greater in the highest R-wave amplitude in the precordial leads, with newly developed giant T-wave inversion, or parallel changes in the negative T waves, or stable ECGs [5]. Newly developed LVH with giant T-wave inversion, and with lead V4 showing the tallest $\mathrm{R}$ wave have been observed [6]. The number of precordial leads with negative T waves $(-0.5 \mathrm{mV}$ or more) was greater in patients with AHCM with giant negative $\mathrm{T}$ waves $(>1.0 \mathrm{mV})$ in the precordial leads; they were also found in more patients than in others, with different distribution of hypertrophy, and no upright $(>1.0 \mathrm{mV}) \mathrm{T}$ waves in the precordial leads were found in patients with AHCM [7]. Patients with giant negative T-wave inversions ( $\mathrm{T}$ wave $>10 \mathrm{~mm}$ ) and high $\mathrm{R}$-wave voltages ( $\mathrm{R}$ wave $>25 \mathrm{~mm}$ ) had more severe apical hypertrophy, although there was incomplete agreement between the ECHO and ECG, and at follow-up there was an increase in the negative T-wave amplitude, with parallel mild increase in the amplitude of $\mathrm{R}$ waves [8] Another study identified the midprecordial leads in most of the patients as the ones revealing the ECG changes in AHCM [9]. Studies of Japanese patients with AHCM detected giant negative $\mathrm{T}$ waves and tall $\mathrm{R}$ waves in lead V5 or high $\mathrm{S}$ wave in $\mathrm{V} 1+\mathrm{R}$ wave in V5 with a decrease in the amplitude of both at long-term follow-up $[10,11]$ The rare occasion of chronic ST-segment elevation and T-wave inversion in the anterolateral leads was reported in one patient with AHCM, who did not have necrosis of the apex or formation of an apical aneurysm [12]. R-wave voltage and T-wave negativity progressively decreased in magnitude on serial ECGs with associated thallium myocardial perfusion defects detected at longterm follow-up [13]. Giant negative $\mathrm{T}$ waves were recorded in the ECG of patients with AHCM who were originally suspected of having acute coronary syndromes [14]. What characterises the entire literature on the ECG in AHCM is its descriptive character, its 'lumping' approach in considering the ECG in patients with AHCM together with other patients with varying left ventricular distribution of hypertrophy, and the absence of mechanistic speculations as to the reasons for which the ECG in such patients appears as it does.

These two cases presented here, in the context of the ECG literature in patients with AHCM, with the quantitative analysis of the ECGs shown in Table 1, is proposed as a 
template to be used in serial ECGs recorded in the long-term follow-up of patients with AHCM, for uniform characterisation of the ECGs of such patients and the changes occurring over time.

Open Access This article is distributed under the terms of the Creative Commons Attribution License which permits any use, distribution, and reproduction in any medium, provided the original author(s) and the source are credited.

\section{References}

1. Fujii J, Saihara S, Sawada H, et al. Distribution of left ventricular hypertrophy and electrocardiographic findings in patient with socalled apical hypertrophic cardiomyopathy. J Cardiogr Suppl. 1985;6:23.

2. Cecchi F, Squillantini G, Zuppiroli A, et al. Correlations between electrocardiographic findings and echocardiographic patterns in 116 patients with hypertrophic cardiomyopathy. G Ital Cardiol. 1986; 16:755.

3. Horita Y, Genda A, Shimizu M, et al. Electrocardiographic and angiographic follow-up of patients with hypertrophic cardiomyopathy. J Cardiol. 1987; 17:259.

4. Horita Y, Genda A, Shimizu M, et al. Serial electrocardiographic and angiographic changes of patients with hypertrophic cardiomyopathy. Jpn Circ J. 1989;53:1327.

5. Nakamura T, Furukawa K, Matsubara K, et al. Long-term followup of electrocardiographic changes in patients with asymmetric apical hypertrophy. J Cardiol. 1990;20:635.

6. Terashima S, Katoh S, Tatsukawa H, et al. Case report: left ventricular apical hypertrophy in progressive limb-girdle muscular dystrophy. Am J Med Sci. 1993;305:166.
7. Usui M, Inoue H, Suzuki J, et al. Relationship between distribution of hypertrophy and electrocardiographic changes in hypertrophic cardiomyopathy. Am Heart J. 1993;126:177.

8. Moro E, D'Angelo G, Nicolosi GL, et al. Long-term evaluation of patients with apical hypertrophic cardiomyopathy. Correlation between quantitative echocardiographic assessment of apical hypertrophy and clinical-electrocardiographic findings. Eur Heart J. 1995;16:210.

9. Kureshi SA, Malik SM, Hyder W. Apical hypertrophic cardiomyopathy in Pakistan: electrocardiographic, echocardiographic and myocardial scintigraphic features. J Pak Med Assoc. 1995;45:117.

10. Koga Y, Katoh A, Matsuyama K, et al. Disappearance of giant negative $\mathrm{T}$ waves in patients with the Japanese form of apical hypertrophy. J Am Coll Cardiol. 1995;26:1672.

11. Hata S, Shikuwa M, Yamasa T, et al. The left ventriculographic pattern and serial electrocardiographic changes in hypertrophic cardiomyopathy patients with giant negative $\mathrm{T}$ waves. Cardiology. 1996;87:365.

12. Penas Lado M, Mosquera Pérez I, Bouzas Zubeldía B, et al. The electrocardiogram in apical hypertrophic myocardiopathy. A case report with unique manifestations. Rev Esp Cardiol. 1999;52:1148.

13. Abinader EG, Sharif D, Shefer A, et al. Novel insights into the natural history of apical hypertrophic cardiomyopathy during longterm follow-up. Isr Med Assoc J. 2002;4:166.

14. Duygu H, Zoghi M, Nalbantgil S, et al. Apical hypertrophic cardiomyopathy might lead to misdiagnosis of ischaemic heart disease. Int J Cardiovasc Imaging. 2008;24:675.

\section{Author's note}

The author of this manuscript has certified that he complies with the Principles of Ethical Publishing in the International Journal of Cardiology. 